\title{
Anomalous Band Formation in Arrays of Terahertz Nanoresonators
}

\author{
Y. M. Bahk, ${ }^{1}$ H. R. Park, ${ }^{1}$ K. J. Ahn, ${ }^{1}$ H. S. Kim,${ }^{1}$ Y. H. Ahn, ${ }^{2}$ Dai-Sik Kim, ${ }^{1, *}$ J. Bravo-Abad, ${ }^{3}$ \\ L. Martin-Moreno, ${ }^{4}$ and F. J. Garcia-Vidal ${ }^{3, \dagger}$ \\ ${ }^{1}$ Center for Subwavelength Optics and Department of Physics and Astronomy, Seoul National University, Seoul 151-747, Korea \\ ${ }^{2}$ Division of Energy Systems Research, Ajou University, Suwon 433-749, Korea \\ ${ }^{3}$ Departamento de Fisica Teorica de la Materia Condensada, Universidad Autonoma de Madrid, E-28049 Madrid, Spain \\ ${ }^{4}$ Instituto de Ciencia de Materiales de Aragon (ICMA) and Departamento de Fisica de la Materia Condensada, \\ CSIC-Universidad de Zaragoza, E-50009 Zaragoza, Spain \\ (Received 15 October 2010; revised manuscript received 25 November 2010; published 5 January 2011)
}

\begin{abstract}
We investigate band formation in one-dimensional periodic arrays of rectangular holes which have a nanoscale width but a length of $100 \mu \mathrm{m}$. These holes are tailored to work as resonators in the terahertz frequency regime. We study the evolution of the electromagnetic response with the period of the array, showing that this dependence is not monotonic due to both the oscillating behavior of the coupling between holes and its long-range character.
\end{abstract}

DOI: 10.1103/PhysRevLett.106.013902

PACS numbers: 41.20.Jb, 42.25.Fx, 42.79.Ag

Band formation in covalent solids is mainly determined by the short-range electronic interaction between their constituent atoms [1]. Because of that, when forming a periodic array of $N$ atoms with period $d$, the bandwidth enlarges when $N$ increases and/or when $d$ is reduced. Fundamental studies on how this picture is translated into the photonic case are scarce and were done in connection with the emergence of band gaps in dielectric photonic crystals [2-4]. In this Letter we carry out a fundamental analysis, both experimental and theoretical, of the photonic band formation in metallic structures. As an illustrating example, we consider as the constituent atom a very elongated rectangular hole perforated on a metal film. A single rectangular hole can support an electromagnetic (EM) resonance for light polarized along the short side of the rectangle [5,6], its spectral location appearing close to the cutoff wavelength of the hole waveguide [7]. Very recently, resonant field enhancements of up to $10^{3}$ have been reported in the terahertz $(\mathrm{THz})$ regime [8]. The transmission properties of periodic arrays of rectangular holes have also been analyzed [9-12], mainly in the context of the phenomenon of extraordinary optical transmission $[13,14]$.

Here we investigate EM band formation through the study of the evolution of the cutoff resonance of a rectangular hole as a 1D array of $N$ resonators separated by a distance $d$ is arranged [see sketch in Fig. 1(a)]. We will show how its EM response presents a nonmonotonic evolution with $N$ and $d$, in contrast to its electronic counterpart. The experimental results are faithfully reproduced by numerical calculations. We also develop a minimal model that clearly relates the anomalous band formation to the long-range character of the interhole EM coupling.

We have fabricated several 1D periodic arrays of rectangular holes, with a fixed length $l=100 \mu \mathrm{m}$ and different widths around $w=200 \mathrm{~nm}$, perforated on $100 \mathrm{~nm}$ thick Au films deposited onto $\mathrm{Si}$ substrates, as shown schematically in Fig. 1(a). The period of the array is varied from 2 to $200 \mu \mathrm{m}$. The arrays were fabricated by electron beam lithography [see Fig. 1(b)]. In our studies, we use the transmitted field through a large aluminum aperture of $1 \mathrm{~mm} \times 1 \mathrm{~mm}$ as a reference field [15]. Because the sample area is limited by the aperture, the number of holes depends on the period, ranging from $N=5$ holes for the case of $d=200 \mu \mathrm{m}$ to $N=500$ for $d=2 \mu \mathrm{m}$. In order to obtain high signal-to-noise ratio, the $1 \mathrm{D}$ structure is cloned 9 times in the $y$ direction with a separation (center
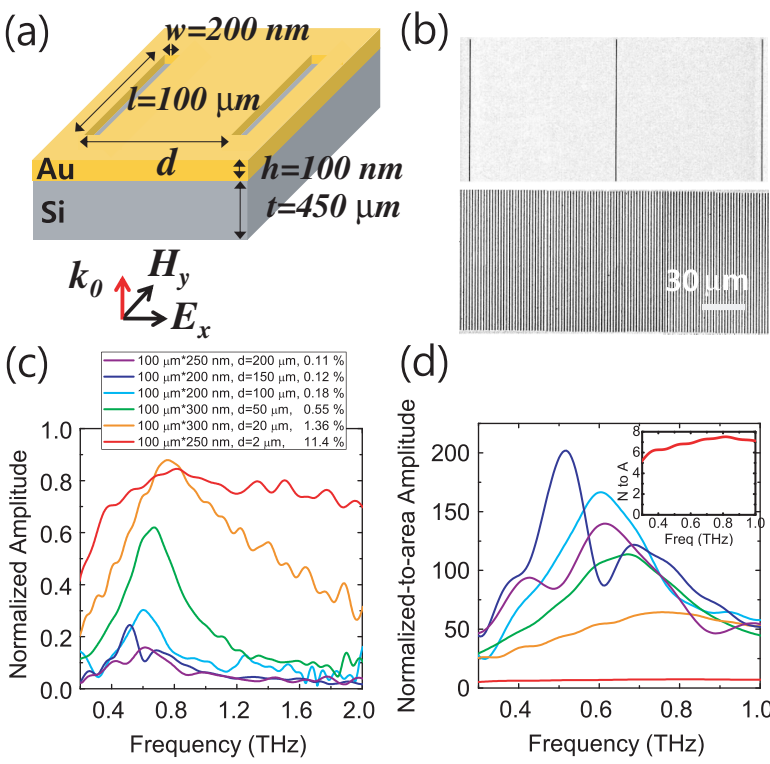

(d)

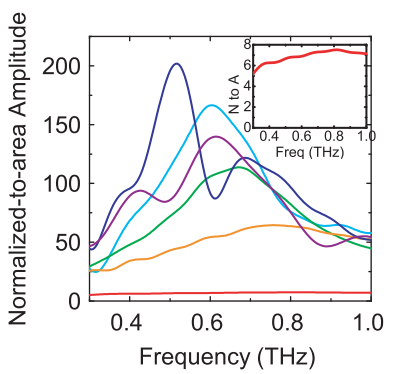

FIG. 1 (color online). (a) Schematic of a periodic array of rectangular holes. The structure is illuminated by normally incident $p$-polarized light. (b) Scanning electronic microscope images of two different periods, $d=100 \mu \mathrm{m}$ and $d=2 \mu \mathrm{m}$. (c) Normalized transmitted amplitudes with varying the periods from $d=200 \mu \mathrm{m}$ to $d=2 \mu \mathrm{m}$. (d) Corresponding normalizedto-area transmitted amplitudes. 
to center) of $110 \mu \mathrm{m}$. We perform $\mathrm{THz}$ time domain spectroscopy to measure the transmission amplitude through the samples. Only the case of normal incidence is considered with the electric field polarized along the short side of the rectangular holes ( $x$ direction), making the EM coupling between rows in the $y$ direction negligible. The transmissivity through the periodic arrays is experimentally characterized by the normalized amplitude, defined as the ratio between the $E$-field amplitudes with and without the holey metal film.

Figure 1(c) renders the normalized transmitted amplitudes for the six samples. As clearly shown in this figure, the magnitude of the transmitted amplitude peak increases as $d$ is decreased and $N$ is increased. Our measurements also show how, for $d=2 \mu \mathrm{m}$ and $N=500$, the transmission band spans over the whole spectral range from 0.2 to $2 \mathrm{THz}$, showing over $80 \%$ normalized transmission amplitude in that frequency interval. Notice that the nanometer scale width of the resonators enables reaching a broadband response, which would be impossible using micron-sized widths [6]. To allow a more direct comparison among the different spectra displayed in Fig. 1(c) (note that, as mentioned, both $N$ and $d$ are varied simultaneously from case to case), we have normalized the transmitted $E$-field amplitude of each sample by the corresponding total area covered by the apertures. As seen in Fig. 1(d), two important observations emerge when using this normalization. On one hand, both the linewidth and the resonant peak position do not show a monotonic behavior as $d$ is changed. Specifically, the transmission spectrum for $d=100 \mu \mathrm{m}$ [see cyan line in Fig. 1(d)] has a similar resonant peak position and linewidth to that of a single isolated rectangular hole. When $d=150 \mu \mathrm{m}$ [blue line in Fig. 1(d)], the transmission spectrum shifts to lower frequencies and displays a reduced linewidth. However, for $d=200 \mu \mathrm{m}$ [violet line in Fig. 1(d)], the linewidth is restored and the resonant peak shifts back towards higher frequencies. On the other hand, Fig. 1(d) clearly shows how for $d<100 \mu \mathrm{m}$ the maximum normalized-to-area transmitted amplitude severely decreases as $d$ is reduced. This is also an unexpected result: one would expect that the normalizedto-area transmittance at resonance should remain almost constant when $d$ varies.

To explain the physical origin of the observations discussed above, we have applied a theoretical coupled-mode formalism based on the modal expansion of the EM fields in the different regions of the structure. A detailed account of this general framework can be found in Refs. [14,16]. This numerical approach can be further simplified if we assume that only the first TE waveguide mode inside the holes is excited during the transmission process. This approximation has proven to be very accurate in the case of subwavelength holes [7]. Thus, within this approach, the modeling of the system reduces to solving a system of linear equations for $E_{\alpha}$ and $E_{\alpha}^{\prime}$ which are the modal amplitudes of the electric field at the input and output sides of the aperture $\alpha$, respectively.
Figure 2 shows our numerical results for the normalizedto-area amplitude as computed for the six 1D finite arrays. As observed in this figure, there is a good quantitative agreement between the theoretical predictions and the corresponding experimental measurements displayed in Fig. 1(d), both in the magnitude of the resonant peak and the evolution with $d$ of its linewidth and position. We emphasize that no fitting parameters are used in this comparison. In order to do analytical work, our theoretical approach can be simplified by imposing that all the holes in the array behave in the same manner, i.e., $E_{\alpha}=E$ and $E_{\alpha}^{\prime}=E^{\prime}$ for all $\alpha$. In this way, the system of linear equations discussed above transforms into one of only two equations for the modal amplitudes of the central hole (labeled from now on as 0 ):

$$
\begin{aligned}
& (G+S-\epsilon) E-G^{V} E^{\prime}=I, \\
& \left(G^{\prime}+S^{\prime}-\epsilon\right) E^{\prime}-G^{V} E=0 .
\end{aligned}
$$

The terms $S=\sum_{\beta \neq 0} G_{0 \beta}$ and $S^{\prime}=\sum_{\beta \neq 0} G_{0 \beta}^{\prime}$ take into account the "collective" reillumination process associated with the propagators $G_{0 \beta}$ and $G_{0 \beta}^{\prime}$ occurring at the two interfaces of the structure. The magnitudes $\epsilon$ and $G^{V}$ are defined as $\epsilon=Y / \tan \left(q_{z} h\right)$ and $G^{V}=Y / \sin \left(q_{z} h\right)$, respectively; $q_{z}=\sqrt{k_{0}^{2}-(\pi / l)^{2}}$ is the propagation constant of the fundamental TE mode inside the holes, whereas $Y=$ $q_{z} / k_{0}$ (with $k_{0}=2 \pi / \lambda$ ) is its corresponding admittance. Finally, $I=(4 \sqrt{2} / i \pi)$ represents the external illumination on the hole array. The extremely good accuracy of this

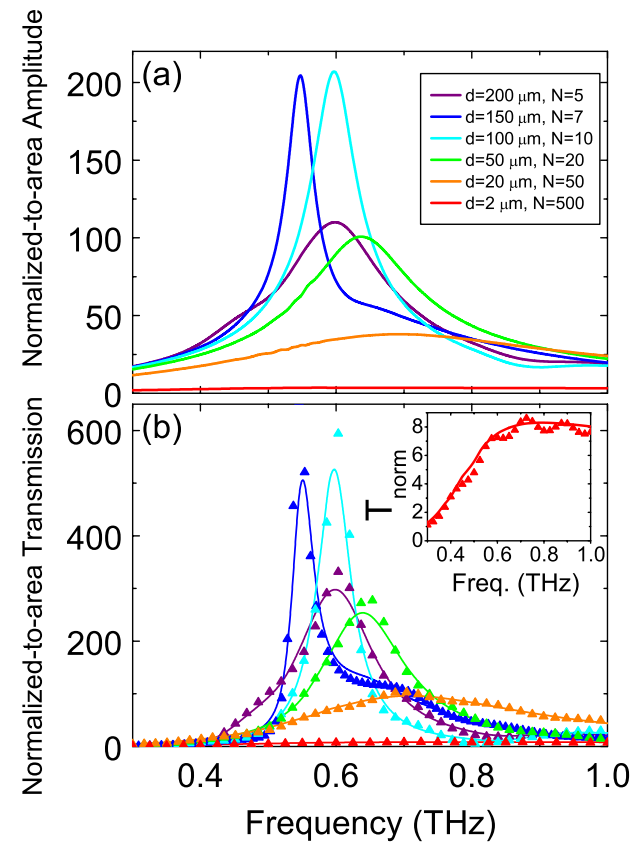

FIG. 2 (color online). Normalized-to-area amplitude (a) and normalized-to-area transmission (b) computed for the six cases of finite array of rectangular holes with $l=100 \mu \mathrm{m}$ and $w=$ $200 \mathrm{~nm}$ considered in Fig. 1. Triangles in (b) render the results obtained from a minimal model (see details in the main text). 
approximation is shown in Fig. 2(b) by comparing their results (triangles) with those emerging from the full calculations (full lines). Notice that, since our theoretical formalism enables a direct calculation of the total transmittance, we have chosen the normalized-to-area transmission as the relevant physical magnitude for this comparison. Moreover, in order to further simplify our analytical study, in the rest of this Letter we consider freestanding films with the same geometrical parameters as those used in the experiments, except for the values of $d$, which have been slightly changed to better illustrate the underlying physical mechanisms. We have checked that the same phenomenology appears in both freestanding samples and holey structures deposited on substrates. For suspended films, $S^{\prime}=S$, allowing us to focus on a single physical magnitude, $S$.

The crucial point is that the system of two linear equations (1) is formally equivalent to that of a single, isolated rectangular hole [see Eq. (1) of Ref. [7]]. The only difference is that the self-illumination term, $G$ for a single hole, now takes into account the reillumination coming from other holes by means of $G+S$. This mapping shows that $S$ can be understood as a "self-energy" that sums up the EM coupling of a given hole with all the other holes. We can then apply some analytical results found for the case of a single hole to a 1D periodic array of rectangular holes. For example, the spectral location of the resonance can be obtained from

$$
2 \operatorname{Re}(G+S)=\frac{|G+S|^{2}-Y^{2}}{Y} \tan q_{z} h,
$$

while the normalized-to-area transmittance at resonance is completely governed by the imaginary part of $(G+S)$,

$$
T_{\text {res }}=\frac{|I|^{2}}{4 \operatorname{Im}(G+S)} .
$$

This last relation illustrates the physical meaning of $\operatorname{Im} S$. For a single rectangular hole, $\operatorname{Im} G$ measures the coupling of the fundamental TE mode with radiative modes in vacuum. The presence of other holes, and the corresponding interference of their radiated fields in the far-field region, makes this quantity to be effectively enhanced $(\operatorname{Im} S>0$, constructive interference) or reduced $(\operatorname{Im} S<0$, destructive interference), depending on $d$. Notice that the linewidth of the transmission resonance is also proportional to $\operatorname{Im}(G+S)$.

Before analyzing the behavior of $S(d)$, it is worth studying how the EM coupling between just two holes, $G_{01}$, depends on its separation $x$; see Fig. 3(a). As expected, both imaginary and real parts present an oscillatory (of period $\lambda$ ) and slow decaying trend, stemming from the behavior of the propagator in real space [16]. The movement in the spectral location of the transmission resonance can be traced back to the evolution of $\operatorname{Re} S(d)$, as rendered in Fig. 3(b), with the help of Eq. (2). Whenever ReS is positive $[d=2$ and $202 \mu \mathrm{m}$ in Fig. 3(b)], the resonance shifts to lower frequencies with respect to the resonance location for a single hole, while moving in the opposite direction when $\operatorname{Re} S$ is negative. Because of the oscillatory behavior of $\operatorname{Re} G_{0 \beta}$ with $\beta$, these shifts are not monotonic functions of $d$.

We now analyze the evolution of $\operatorname{Im} S(d)$, which governs the peak transmittance and its linewidth, as a function of $N$. When $N$ is small and $d \ll(l, \lambda)$, we obtain $\operatorname{Im} G_{0 \beta}(\beta d \rightarrow 0) \approx \operatorname{Im} G$. This implies that $\operatorname{Im} S$ presents a linear scaling with $N, \operatorname{Im} S \approx(N-1) \operatorname{Im} G$, as observed in the case $d=2 \mu \mathrm{m}$ displayed in Fig. 3(c). Correspondingly, as predicted by Eq. (3), the normalized-to-area transmittance is reduced by a factor $N$ with respect to that of a single hole [see the corresponding maximum of transmission $T_{\max }$
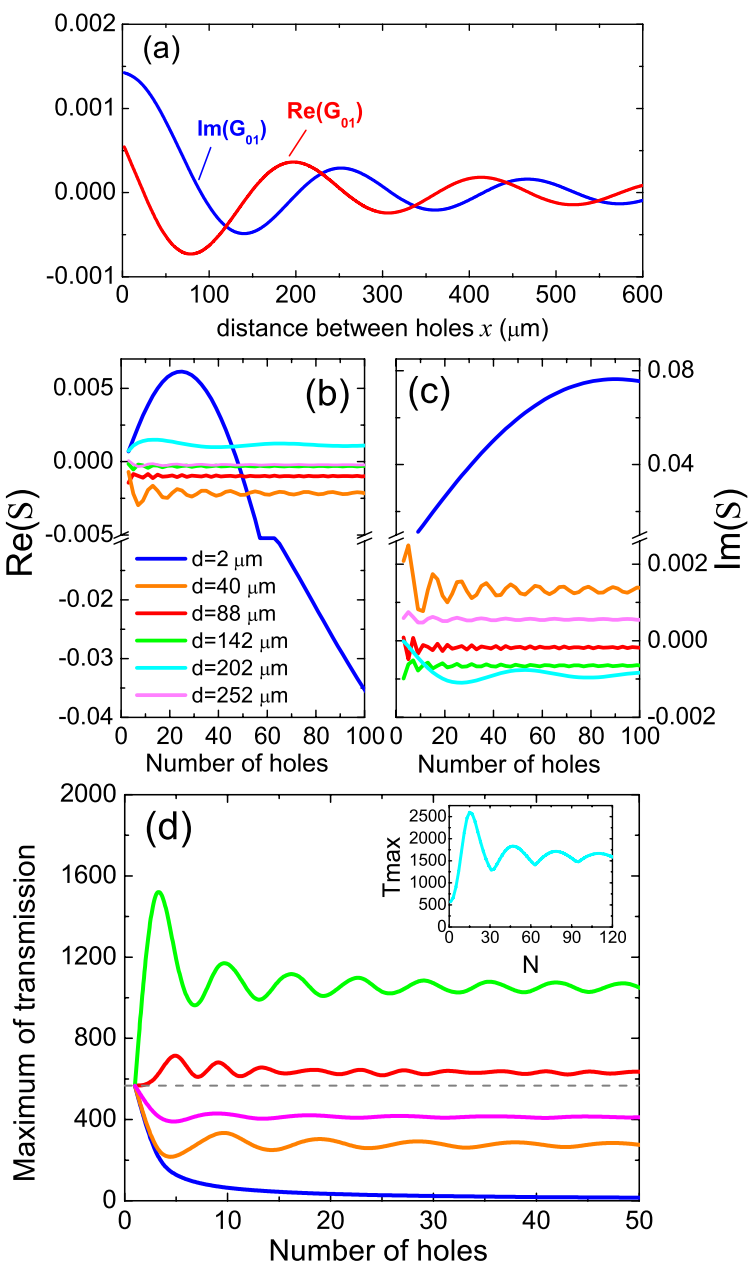

FIG. 3 (color online). (a) Imaginary and real parts (shown as blue lines and red lines, respectively) of the EM coupling between two holes, $G_{01}$ (see main text for details on this magnitude). Panels (b) and (c) render the computed real and imaginary parts, respectively, of $S$ versus the number of holes $N$. All the magnitudes shown in this figure are evaluated at the resonance frequency of a single hole, $f_{R}=1.43 \mathrm{THz}\left(\lambda_{R}=\right.$ $209 \mu \mathrm{m})$. (d) Maximum normalized-to-area transmission $T_{\max }$ versus the number of apertures computed for the different values of $d$ considered in (b) and (c). Inset displays the case corresponding to $d=202 \mu \mathrm{m}$. Dashed gray line in main figure corresponds to the value of $T_{\max }$ for a single isolated hole. 
versus $N$ in Fig. 3(d)], and a very broad transmission spectrum is obtained. Notice that, at resonance, the intensity of the $E$ field at the apertures, $|E|^{2}$ or $\left|E^{\prime}\right|^{2}$, is proportional to $T_{\max }^{2}$ [7]. Therefore, the mentioned broadband response also yields to a broadband enhancement of the corresponding $E$ field.

As $d$ is increased but still $d<\lambda / 2, \operatorname{Im} G_{0 \beta}$ is still positive for the first neighbor interaction, resulting in a short-ranged positive $\operatorname{Im} S$ [see case $d=40 \mu \mathrm{m}$ in Fig. 3(c)]. As shown in Fig. 3(d), this short-range interaction yields to a rapid saturation of $T_{\max }$ as a function of $N$. However, when $d \approx \lambda / 2$ [the case $d=88 \mu \mathrm{m}$ in Fig. 3(c)], $\operatorname{Im} S$ is close to zero, and therefore $T_{\max }$ and, consequently, the corresponding linewidth, are very similar to those of a single hole [see Fig. 3(d)]. Eventually, for even larger values of $d, \operatorname{Im} S$ can be negative $(d=142 \mu \mathrm{m}$ and $d=202 \mu \mathrm{m} \approx \lambda$ ), leading to a reduction in their radiative coupling and to an enhanced transmittance at maximum, i.e., to a sharper spectrum. As observed in Fig. 3(d), the long-range interhole coupling occurring when $d \approx \lambda$ produces large amplitude oscillations in the evolution of $T_{\max }$ versus $N$. In addition, notice that in this case, huge enhancements of $|E|^{2}$ and $\left|E^{\prime}\right|^{2}$ are obtained within a very narrow range of frequencies. This narrowing effect occurring at $d \approx \lambda$ has been previously described in arrays of resonators and ascribed either to the formation of lattice resonances [17] or to coherent coupling between the elements [18]. In our case, further increase in $d$ results in $\operatorname{Im} S$ being positive again, with a slightly reduced transmittance and broader spectrum [see the case $d=252 \mu \mathrm{m}$ in Figs. 3(b) and 3(d)].

Finally, some comments on the comparison of the evolution of $T_{\max }$ versus $N$ shown in Fig. 3(d) with the case of 1D arrays of slits [19] are pertinent. The origin of the significant differences between the two cases lies in the fundamentally different physical origin of the EM resonances we consider here (cutoff resonances) with respect to both conventional Fabry-Perot and surface EM resonances appearing in 1D arrays of slits. In particular, as our findings have shown, the large transmission cross section characterizing rectangular holes with extremely large aspect ratio enables accessing novel interhole coupling regimes appearing when the period is varied. Thus, it is expected that similar phenomena as those reported in this Letter can be observed in periodic arrangements of different classes of resonators displaying EM resonances characterized by very large cross sections.

In conclusion, we have shown that the electromagnetic coupling between nanoresonators forming a periodic array presents a much more complex behavior than the electronic interaction between atoms forming a solid. In addition, from an applied point of view, our results provide a complete landscape of electric field enhancements attainable in the considered structures, which could help in engineering their response. In particular, these results show great promise for a wide spectrum of important applications benefiting from broadband electric field enhancements, such as nonlinear frequency-mixing and sensing devices or thin-film photovoltaics.

The experimental work was supported by the Korea Science and Engineering Foundation (KOSEF) (SRC, No. R11-2008-095-01000-0), the Korea Research Foundation (KRF), KICOS (GRL, K20815000003), Hi Seoul Science/Humanities grant funded by the Korea government (MEST) (No. 2009-0071309), the Seoul R\&BD Program (10543), and Seoul Scholarship Foundation. The theory work was funded by the Spanish Ministry of Science and Innovation under Projects No. MAT200806609-C02, No. CSD2007-046-NanoLight.es, and by Grant No. RyC-2009-05489.

*dsk@phya.snu.ac.kr †j.garcia@uam.es

[1] N.W. Ashcroft and N. D. Mermin, Solid State Physics (Sounders, Philadelphia, 1976).

[2] E. Lidorikis, M. M. Sigalas, E. N. Economou, and C. M. Soukoulis, Phys. Rev. Lett. 81, 1405 (1998).

[3] A. Yariv, Y. Xu, R. K. Lee, and A. Scherer, Opt. Lett. 24, 711 (1999).

[4] M. Bayindir, B. Temelkuran, and E. Ozbay, Phys. Rev. Lett. 84, 2140 (2000).

[5] A. Degiron, H. J. Lezec, N. Yamamoto, and T.W. Ebbesen, Opt. Commun. 239, 61 (2004).

[6] J. W. Lee, M. A. Seo, D. H. Kang, K. S. Khim, S. C. Jeoung and D. S. Kim, Phys. Rev. Lett. 99, 137401 (2007).

[7] F. J. Garcia-Vidal, E. Moreno, J. A. Porto, and L. MartinMoreno, Phys. Rev. Lett. 95, 103901 (2005).

[8] M. A. Seo, H. R. Park, S. M. Koo, D. J. Park, J. H. Kang, O. K. Suwal, S. S. Choi, P. C. M. Planken, G. S. Park, N. K. Park, Q. H. Park, and D.S. Kim, Nat. Photon. 3, 152 (2009).

[9] K. J. Klein Koerkamp, S. Enoch, F. B. Segerink, N. F. van Hulst, and L. Kuipers, Phys. Rev. Lett. 92, 183901 (2004).

[10] D. X. Qu, D. Grischkowsky, and W. L. Zhang, Opt. Lett. 29, 896 (2004).

[11] A. Degiron and T. W. Ebbesen, J. Opt. A 7, S90 (2005).

[12] Z. Ruan and M. Qiu, Phys. Rev. Lett. 96, 233901 (2006).

[13] T. W. Ebbesen, H. J. Lezec, H. F. Ghaemi, T. Thio, and P. A. Wolff, Nature (London) 391, 667 (1998).

[14] F. J. Garcia-Vidal, L. Martin-Moreno, T. W. Ebbesen, and L. Kuipers, Rev. Mod. Phys. 82, 729 (2010).

[15] J.S. Kyoung, M. A. Seo, H.R. Park, K. J. Ahn, and D. S. Kim, Opt. Commun. 283, 4907 (2010).

[16] J. Bravo-Abad, F. J. Garcia-Vidal, and L. Martin-Moreno, Phys. Rev. Lett. 93, 227401 (2004).

[17] G. Vecchi, V. Giannini, and J. Gomez Rivas, Phys. Rev. Lett. 102, 146807 (2009).

[18] V. A. Fedotov, N. Papasimakis, E. Plum, A. Bitzer, M. Walther, P. Kuo, D. P. Tsai, and N. I. Zheludev, Phys. Rev. Lett. 104, 223901 (2010).

[19] A. I. Fernandez-Dominguez, F. J. Garcia-Vidal, and L. Martin-Moreno, Phys. Rev. B 76, 235430 (2007). 